\title{
Esculape africain
}

\section{N. Benseddik}

\section{OpenEdition}

Journals

Édition électronique

URL : http://journals.openedition.org/encyclopedieberbere/2001

DOI : 10.4000/encyclopedieberbere.2001

ISSN : 2262-7197

\section{Éditeur}

Peeters Publishers

\section{Édition imprimée}

Date de publication : 1 août 1997

Pagination : 2691-2698

ISBN : 2-85744-948-8

ISSN : 1015-7344

\section{Référence électronique}

N. Benseddik, «Esculape africain », Encyclopédie berbère [En ligne], 18| 1997, document E41, mis en ligne le 01 juin 2011, consulté le 24 septembre 2020. URL : http://journals.openedition.org/ encyclopedieberbere/2001; DOI : https://doi.org/10.4000/encyclopedieberbere.2001

Ce document a été généré automatiquement le 24 septembre 2020

(c) Tous droits réservés 


\title{
Esculape africain
}

\author{
N. Benseddik
}

\section{Implantation du culte}

Comme celui des Cereres, d'Hercule ou de Genii municipaux, le culte d'Esculape a joui d'une faveur particulière en Afrique. Le culte guérisseur n'y a pourtant pas rencontré un succès égal partout : l'implantation a été inégale non seulement d'une province à une autre, mais aussi à l'intérieur d'une même province, en relation avec des circonstances locales, tant géographiques que sociales. Un regard jeté sur la carte du culte permet de constater que les traces laissées par les témoignages de dévotion à l'adresse d'Esculape et d'Hygie, sa fille, diminuent vers l'ouest, constituant trois groupes nettement individualisés: les vieilles cités berbéro-puniques, les agglomérations établies sur les voies de pénétration de la romanisation et les stations thermales, certains lieux appartenant à deux ou trois groupes à la fois. En Proconsulaire, Esculape apparaît plutôt seul ou avec Caelestis, Hygie étant rarement mentionnée. A l'exception du camp de Gholaïa, sur le limes de Tripolitaine, le culte y revêt un aspect civil. Au contraire, la Numidie est la province du culte commun d'Esculape et Hygie. Les deux divinités y ont bénéficié d'un culte franchement militaire dans les camps et les villes de garnison, thérapeutique dans les thermes. En Maurétanie Césarienne, en dehors de la capitale, la présence des dieux guérisseurs reste insignifiante et souvent hypothétique, les auxiliaires ne paraissant pas avoir partagé la ferveur de leurs collèges de la Illa Augusta pour Esculape et Hygie. En Tingitane, les deux sculptures de Banasa et Volubilis ne peuvent contredire l'impression que le culte a rencontré peu de succès dans la région. Un parallèle entre la diffusion géographique du culte d'Esculape et celle du culte de Saturne souligne le caractère élitiste du premier. En effet, l'implantation inégale du culte du maître du panthéon africain dépend, selon $\mathrm{M}$. Le Glay, à la fois d'une situation socio-économique caractérisée par les formes de vie sédentaires des agriculteurs-éleveurs et de l'influence gréco-romaine, la densité des sanctuaires variant donc en fonction de celle de la population rurale et en proportion inverse du degré de romanisation ; à l'opposé, l'implantation du culte d'Esculape est en étroite relation avec la densité de la population urbanisée et le degré de romanisation. 
2 A l'inégale implantation géographique du culte, s'ajoutent des disparités sociales significatives. Les fidèles d'Esculape et d'Hygie, en Afrique, forment quatre grands groupes dont le plus important est celui des fonctionnaires, des militaires, des agents divers de l'administration impériale ; cette catégorie, dominée par les gouvernements et les militaires, donne un cachet officiel et élitiste au culte en Numidie militaire et en Césarienne, l'armée, la III légion en particulier, se révélant un vecteur essentiel de diffusion du culte guérisseur dans la région. Le deuxième groupe est celui de la bourgeoisie municipale et provinciale (magistrats, prêtres des cités, patrons). En dehors des flamines, la part prise par ces notables municipaux dans le culte guérisseur apparaît aussi modeste que celle des villes. Les prêtres constituent une catégorie dont le petit nombre nous parait imputable à la seule indigence documentaire. Encore plus modeste est le groupe des esclaves et des affranchis venus de Grèce et d'Orient; contrairement à ce qui se passe dans d'autres régions de l'empire, cette catégorie de fidèles ne paraît avoir joué aucun rôle dans l'implantation du culte en Afrique. L'absence d'une catégorie traditionnellement respective au message du dieu d'Epidaure - les petites gens - ne manquent pas de surprendre. Alors qu'on présente souvent le culte d'Esculape comme celui des masses et non celui de l'élite, des couches inférieures plutôt que des classes supérieures, des gens incultes et non des personnes cultivées, la documentation africaine, compte tenu de son caractère limité, prouve exactement l'inverse: en Afrique, les petites gens ne font pas partie des adeptes d'Esculape. Ce dernier fait donc partie de ces divinités auxquelles fonctionnaires et soldats adressaient leurs hommages, mais qui ne sont pas, en règle générale, devenues populaires dans les provinces qu'administraient ces fonctionnaires et où ces soldats tenaient garnison. N'est-il pas naturel, alors, de penser que l'immense majorité silencieuse a boudé le dieu guérisseur gréco-romain parce qu'elle réservait sa dévotion à d'autres divinités de la santé ?

\section{Un dieu à plusieurs facettes}

3 La nature des témoignages du culte comme leur répartition font apparaître Esculape sous divers aspects.

\section{Le dieu gréco-romain}

4 Fils d'Apollon et d'une mortelle, Coronis, Asklépios a d'abord été vénéré en tant que héros. En tant que dieu, il n'a été reçu par tous les Grecs que relativement tard puisque la plus ancien document est une inscription athénienne commémorant l'arrivée de dieu à Athènes en $420 \mathrm{av}$. J.-C. C'est à partir d'Epidaure et à une date relativement tardive qu'Asklépios a commencé à conquérir la Grèce. L'époque hellénistique est celle de la reconnaissance universelle du dieu et de l'édification de temples dans toutes les grandes cités, mais son admission à Rome, en 291 av. J.-C. constitue le point de départ de l'ascendance du culte en Occident. La popularité du dieu guérisseur dans l'armée a fortement contribué à l'expansion de son culte, puisque les soldats l'ont emmené dans toutes les régions dominées par Rome, jusqu'aux extrémités du monde habité. En Afrique, les témoignages du culte rendu à Esculape et à Hygie se concentrent dans les vieilles cités berbéro-puniques, les agglomérations établies sur les voies de pénétration de la romanisation et les stations thermales. 


\section{a) Les vieux centres pré-romains}

5 En Proconsulaire, le culte gréco-romain est attesté à Carthage, Dj. Bou Kornein, Gammarth, Utique, Curubis, Asadi, Hadrumetum, Thysdrus, Gigthis, Oea, Lepcis Magna, $\mathrm{Hr}$ Berjeb, Hr Chett, Thuburbo Maius, Thignica, Hr Bir el Afu, Thugga, Vchi Maius, Vazi Saura, Mactar, Althiburos, Hippo Regius, Madauros, Thubursicu Numidarum, Calama et Theveste.

6 En Numidie, le culte a laissé des traces peut-être à Cirta, si la statue qu'abrite le musée, mais dont on ne connaît pas le lieu de provenance, représente bien Esculape.

7 En Maurétanie Césarienne, Caesarea a réservé un culte officiel à la divinité. En Tingitane, enfin, Volubilis ne doit sa présence sur la carte du culte qu'à une statuette qui peut représenter aussi bien Esculape jeune qu'Apollon.

\section{b) Les voies de pénétration}

8 Outre les villes anciennes déjà mentionnées, qui sont également situées sur des voies de communication, de nombreux autres lieux de culte suivent les axes de pénétration de la romanisation. C'est, en Proconsulaire, Maxula et Gholaïa; en Numidie, Rusicade, Sila, Cuicul, Mascula, Thamugadi, Lambaesis, Lambiridi, Zaraï, El Gahra et Castellum Dimmidi ; en Césarienne, Auzia, Rapidum; en Tingitane, Banasa.

\section{c) Les stations thermales}

9 C'est encore le dieu gréco-romain et sa fille que nous retrouvons comme protecteurs de diverses stations thermales de Proconsulaire et de Numidie. Dans la première, Aquae Persianae, Dj. Oust, Hammam Djedid, Hammam Zriba et Aquae Aptuccensium ont livré dédicaces ou statues attestant leur présence. En Numidie, le couple guérisseur n'apparaît qu'à Aquae Flauianae.

Bien que le dieu de la médecine honoré dans ces trois catégories de lieux de culte l'ait été sous son aspect importé, son culte y présente des différences, non seulement d'une province à une autre mais également à l'intérieur d'une même province. A Carthage, à Lepcis Magna, Thubursicu Numidarum ou Lambèse, par exemple, vénéré seul ou avec Hygie, Esculape a su rester fidèle à ses origines gréco-romaines. La découverte à Gammarth d'un autel dédié Aesculapio ab Epidauro, au II siècle, donne une teinte d'hellénisme au culte guérisseur en Afrique : le dédi-cant, prêtre de Cybèle et d'Attis, éprouve le besoin de préciser qu'il s'adresse à l'Esculape d'Epidaure, l'Esculape " authentique ", sans doute pour le distinguer de l'Esculape "commun » qui doit lui paraître trop africanisé. Toujours gréco-romain, le culte guérisseur rendu dans une cité par des membres de l'aristocratie municipale, de l'administration impériale ou municipale au dieu seul, se révèle néanmoins différent de celui qui l'associait à Hygie dans les thermes et les stations thermales ou de celui qui suscitait la ferveur des officiers de la Illa Augusta. Riche de tout un arrière-plan culturel gréco-romain, cet Esculape prend place parmi ces divinités gréco-romaines qui étaient des divinités d'une classe, celle qui dans la ville, aux assemblées provinciales, auprès du gouverneur, avait le pouvoir. En Césarienne, où le culte ne doit rien à l'armée mais reste nettement officiel, et en Tingitane, le hasard des découvertes ne peut justifier la minceur des témoignages ni masquer l'impression d'échec: quels cultes traditionnels, quelles divinités maures y ont empêché son implantation? 


\section{Le dieu phénico-punique}

11 Sur le revers d'une monnaie en bronze de Béryte, datée d'Elagabale, Eshmun, debout, de face, juvénile et nu, regarde à droite, les cheveux coiffés en chignon ;le bras droit est levé, la main droite ouverte à hauteur de la tête. Une draperie, posée sur son épaule gauche, s'enroule autour du bras étendu au-dessus de l'un des dragons ailés, à tête cornue et barbue, qui se dressent symétriquement à ses côtés. Un aureus attribué par $\mathrm{E}$. Babelon à l'Afrique et à Septime Sévère offre une image très proche : sous le fronton triangulaire d'un temple distyle, considéré comme celui d'Eshmun à Carthage, se dresse le dieu, debout de face, imberbe et entièrement nu ; il s'appuie de la main droite sur un bâton autour duquel est enroulé un serpent, tandis qu'à ses pieds, symétriquement à droite et à gauche, se trouvent deux serpents à la tête cornue enroulés et dressés sur leurs anneaux. Alors que l'influence gréco-romaine est évidente sur l'exemplaire africain, l'image du dieu phénicien sur la monnaie de Béryte, encore sous le règne d'Elagabale, est fidèle à la pure tradition phénicienne. C'est en effet à Esculape que la divinité représentée sur l'aureus africain doit ses formes épaisses et le bâton au serpent, mais c'est à Eshmun qu'elle emprunte son aspect juvénile et les deux serpents ou dragons d'Astarté. Cet exemple remarquable de fusion iconographique entre la divinité phénicienne et le dieu grec est un premier indice de la nature complexe d'Esculape africain.

Le culte carthaginois s'intègre dans un héritage culturel qui remonte non seulement au Baal de Sidon, dieu poliade et protecteur individuel à la fois, mais, plus loin, au « dieuhuile » d'Ugarit. D'anciennes pratiques tant magiques que médicales expliqueraient le glissement sémantique de smn " huile » à " celui qui oint " puis à "guérisseur »; nom commun, 'smn serait devenu une épithète du dieu sidonien puis un nom divin à part entière, faisant d'Eshmun un dieu guérisseur, identifié ensuite avec Asklépios, le divin médecin grec. Parmi les dieux qui régnaient sur le panthéon de Carthage, Eshmun apparaît en troisième position, après Baal Hammon et Tanit Péné Baal. Selon Apulée (Floride, 18), l'Esculape punique était le protecteur spécifique de Carthage, le Baal poliade garant de son bien-être et de celui de ses habitants. Le grand nombre de théophores puniques composés à partir de son nom et fournis aussi bien par l'épigraphie punique que par l'épigraphie latine africaine, nous permet d'apprécier le degré de vénération vouée à Eshmun par les Carthaginois. Si l'on admet que les théophores sont l'expression d'une dévotion plutôt privée et familiale, on ne peut que se réjouir de l'importance d'un tel répertoire pour l'analyse des rapports qu'entretenaient avec cette divinité les fidèles qui plaçaient leurs nouveau-nés sous sa protection éternelle. D'après ce répertoire, Eshmun apparait à Carthage comme une divinité "secourable ", "qui délivre ", " qui garde », " conserve », autant d'épithètes qui conviennent à un dieu bienfaisant, un dieu de la santé. Ses dévots se proclament ses " serviteurs » ou ses « servantes », ses " protégés » ou " clients ", se mettent « dans ses mains». Ce corpus n'est pas spécifique d'Eshmun car ces actions secourables appartiennent aussi à d'autres divinités, mais leur concentration et leur variété, impressionnantes dans le cas de cette divinité, ne trouvent pas de parallèle dans le panthéon punique (Melqart et Astarté compris). Eshmun apparaît donc bien comme le plus disponible et le plus attentif aux requêtes des hommes. On a dénombré plus de 700 théophores carthaginois comportant le nom d'Eshmun, une vingtaine constituée de 
formations nominales différentes. Ils sont en nombre beaucoup plus réduit dans le domaine néo-punique mais il sont, géographiquement, bien répartis: Carthago, Hadrumetum, Teboursouk, Mactar, Calama, Cirta, Caesarea; cette diminution ne résulte pas d'un déclin de popularité mais d'un processus syncrétique avec Esculape qui ne devrait pas empêcher de deviner la présence sous-jacente de l'ancien culte punique. Six inscriptions carthaginoises mentionnent le temple d'Eshmun et ses «serviteurs ", mais c'est surtout Appien (Historia romana, VIII, 130-131), dans son récit de la chute de Carthage, qui nous renseigne sur le lieu de culte: situé sur l'acropole, il est vaste, entouré d'une enceinte et servait à des réunions; son toit, probablement en terrasse, pouvait porter plusieurs centaines d'hommes et dominait de haut les alentours.

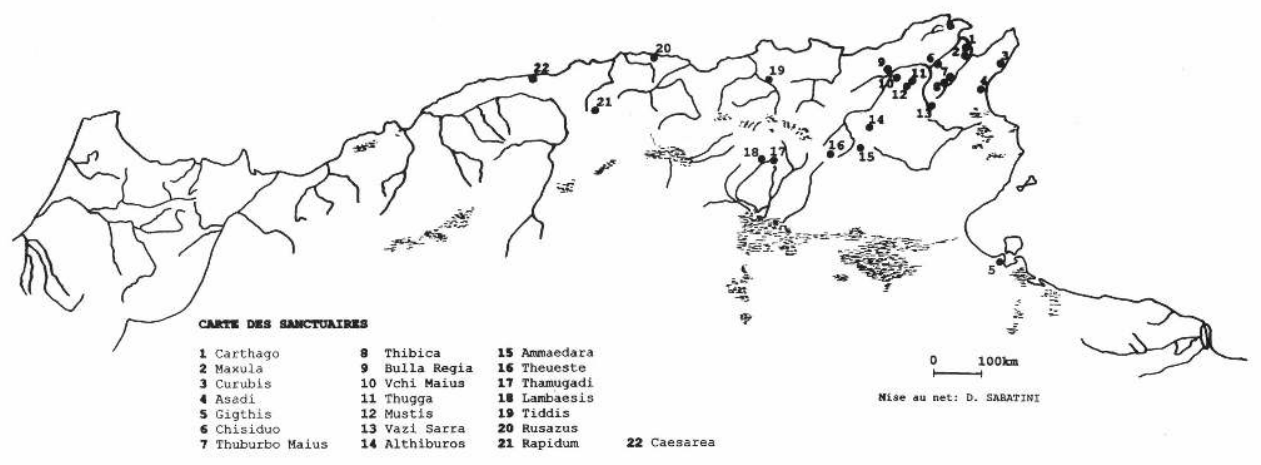

13 C'est dans ce temple que s'étaient réfugiés, en - 146, Asdrubal et d'autres Carthaginois et que se joua le dernier acte de la tragédie de la métropole punique.

\section{Le dieu berbéro-romain}

Une des sept divinités trônant sur un bas-relief découvert à Béja, en Tunisie, un uolumen dans la main droite et un bâton autour duquel s'enroule un serpent, dans la gauche, porte le nom de Macurgum. Construit sur le trilitère libyque mqr (moqran: grand), ce nom, dont la racine est celle d'un certain nombre d'ethniques berbères, se retrouve, avec une inversion du $\mathrm{c}$ et du g, dans le Magu(r)cum Fortunatus d'une stèle à Saturne de Hr es-Srira; on retrouve par ailleurs dans les croyances kabyles un génie nommé Maqur. A gauche de Macurgum, est assise Vihinam, une déesse couverte d'une chape d'écaillés ou de plumes, tenant un forceps (ou un gâteau-couronne), un enfant à ses pieds.

La présence du uolumen et du bâton au serpent, attribut caractéristique de la divinité gréco-romaine de la santé, dans les mains de Macurgum, éclaire sur la fonction guérisseuse de ce dernier, oblige à s'interroger sur les relations de ces deux divinités et surtout à évaluer la place de la composante berbère dans un culte qui paraissait jusque là fidèle à ses origines gréco-romaines et dont on vient d'apercevoir l'arrière-plan culturel phénico-punique. Il est facile d'imaginer que des divinités - certaines d'importation plus ancienne, à savoir phénicienne, d'autres indigènes -, avaient en charge les problèmes de santé des Africains avant l'introduction d'Aesculapius dans le 
pays. Là, comme dans bon nombre d'autres provinces de l'empire, d'ailleurs, les réalités indigènes se sont transformées au contact de Rome sans que les progrès de la romanisation aient signifié leur disparition. Ainsi à la première forme de syncrétisme connue - le syncrétisme libyco-punique - s'est ajoutée une interprétation des cultes libyques, libyco-punique et punique sous une forme romaine, ce qui donne une idée de la difficulté de reconnaître ces différents niveaux dans la personnalité d'Esculape africain. Outre son nom libyque de Macurgum, la divinité guérisseuse du bas-relief de Béja nous livre une image qui ne manque pas d'originalité. Si les sculptures africaines d'Esculape ne portent pas les marques d'un quelconque emprunt iconographique indigène, à l'inverse, un détail du bas-relief de Vaga illustre parfaitement l'assimilation du dieu importé par une divinité berbère de la santé: l'emprunt des attributs caractéristiques de la divinité gréco-romaine de la santé. Objet d'un culte dans l'Afrique berbère, précarthaginoise, le serpent a très bien pu jouer, en Afrique, le même rôle qu'en Phénicie dans le processus d'identification d'un dieu indigène guérisseur à un dieu guérisseur importé. De même que des aspects essentiels d'Eshmun ont facilité son assimilation à Asklepios, il faudra bien admettre que des éléments symboliques de l'imaginaire collectif africain, dont le serpent, attachés à Macurgum, dieu libyque de la santé, ont concouru à le remplacer par le dieu gréco-romain Aesculapius; la présence de l'attribut par excellence de celui-ci dans la main gauche de Macurgum atteste déjà l'identité de fonction entre les deux divinités. G. Camps se posait, à juste titre, la question de savoir si le voisinage de Vihinam était intentionnel ou le fruit du hasard. En fait, rien ne serait plus logique que de placer côte à côte le dieu de la guérison et la déesse de l'enfantement et de la fécondité. Le couple Macurgum/Vihinam pourrait bien être le pendant indigène de celui d'Esculape/Hygie.

Ce monument illustre bien ce que J. Toutain nommait «la greffe religieuse » d'une divinité importée sur une divinité indigène et que nous considérons comme une véritable interpretatio africana. L'image qu'il nous livre est le fruit d'une assimilation religieuse favorisée par des contacts précoces, dans ce centre de commerce, avec la civilisation romaine importée par des marchands italiques dès l'époque de la guerre de Jugurtha. C'est aussi la démonstration d'une fusion intime à la fois entre les cultes puniques et libyques et entre la tradition berbéro-punique et la culture gréco-romaine. Les cultes libyques, on peut le constater, gardent toute leur vivacité malgré la multiplicité des apports et l'intense pression des influences tant phéniciennes que gréco-romaines : les dédicants portent les tria nomina, mais s'adressent à leurs divinités ancestrales, habillées d'éléments empruntés à des cultes importés. Ni puniques, ni grecs, ni romains, les dieux de M. Aemilius Ianuarius et de $Q$. Aelius Félix ne sont pourtant plus tout à fait berbères. Le monument semble avoir appartenu à un petit sanctuaire rural où nos divinités indigènes, assimilées aussi bien à des dieux puniques qu'à des déités gréco-romaines, recevaient des hommages émanant, comme celui-ci, d'humbles fidèles à demi romanisés qui leur demeuraient attachés, des siècles après l'arrivée de cultures étrangères. Tout en empruntant à l'iconographie hellénique d'Esculape certains traits, l'image de Macurgum reste néanmoins attachée au style de ce bas-relief, style qu'on retrouve sur des monuments découverts dans le voisinage et à Hr Ramdane : même costume, même coiffure, hiératisme identique des personnages représentés de face. Une telle parenté iconographique atteste l'existence de panthéons locaux ou régionaux vénérés dans d'autres sanctuaires du même type. interpretatio africana constatée, par exemple à Béja, répond, dans le sens inverse l' 
interpretatio romana, à savoir la fusion de la divinité gréco-romaine avec une ou plusieurs divinités indigènes guérisseuses dont les noms sont rarement connus; ce processus produit ainsi une divinité berbéro-romaine dans laquelle il est rarement possible de distinguer l'élément importé de l'élément autochtone et qui devient ainsi, malgré un nom latin et un habillage gréco-romain, propre au panthéon africain. Paradoxalement, la dédicace de Gammarth "Aesculapio ab Epidauro" prouve à la fois l'africanisation du dieu grec de la santé et le caractère inachevé de la fusion de celui-ci avec Eshmun, Macurgum ou une autre divinité berbère guérisseuse : chacun, dans cette ambiance générale de syncrétisme, était libre de se référer à la divinité dont il se sentait le plus proche. Quand il n'est pas grec, Esculape peut être en Afrique soit le produit de la fusion entre le dieu importé et le dieu phénicien, soit celui du mélange entre le premier et des génies berbères pourvus de pouvoirs guérisseurs, soit encore un mélange des trois, l'Eshmun punique assumant lui-même l'héritage de croyances antérieures à son arrivée. A Carthage, par exemple, que dominait le temple du dieu punique, le premier type d'assimilation a dû se produire souvent, tandis que dans la région de Béja-Chemtou, protégée par Macurgum, c'est plutôt le second qui a permis l'élaboration d'une nouvelle divinité guérisseuse. La présence conjointe, en Proconsulaire tout au moins, de trois divinités de la santé sous l'appellation ou l'image d'Aesculapius -celle d'Epidaure, la punico-romaine et la berbéro-romaine - signifie qu'une certaine concorde, qui repose sur la reconnaissance implicite des différences, existe aussi bien entre les hommes qu'entre les dieux. Pourtant, quel que soit son degré d'achèvement, le phénomène syncrétiste des cultes thérapeutiques en Afrique montre ses limites dans l'incontestable persistance des éléments religieux traditionnels africains, leur renforcement à certains moments et même leur résurgence après une période de sommeil, probablement parce que le syncrétisme n'a pas entraîné en bloc toute la population, mais un ensemble diversifié, voire contrasté, d'attitudes religieuses propres à chaque groupe social. Qu'on attribue ces phénomènes syncrétistes à des tentatives imposées d'en haut, par Rome, dans le cadre d'une politique d'intégration des élites, ou au désir mimétique d'une partie de la population africaine, on comprend mieux la minceur de la documentation relative à l'Esculape africain, les initiatives de personnages officiels s'exprimant au nom de la communauté constituant l'essentiel de notre matériel. Le silence épigraphique de larges couches de la population dans la documentation relative au culte d'Esculape est avant tout l'expression d'une diffusion sociale élitiste du culte en Afrique ; il confirme aussi le rôle déterminant de la religion dans l'intégration de cette région de l'empire dans la romanité, certaines classes ayant soutenu et répandu l'idéologie du conquérant. Ainsi, malgré certaines réussites du syncrétisme africain, on est tout de même forcé de constater qu'une véritable fusion des héritages religieux romain et africain ne s'est opérée que pour une minorité de la population de l'Afrique romaine.

\section{BIBLIOGRAPHIE}

TOUTAIN J.,Les cultes paiens dans l'Empire romain, t. I, Paris, 1905, p. 330s.q. 
EDELSTEIN, E.-J.-L., Asclepius, a collection and interpertation of the testimonies, I-II, Baltimore, 1945.

MERLIN A., C.R.A.I., 1947, p. 335-371.

PICARD G.-Ch., Les religions de l'Afrique antique, Paris, 1954.

CAMPS G.,L'inscription de Béja et le problème des Dii Mauri, R. Afr., 98, 1954, p. 233-258 ; Qui sont les Dii Mauri, Ant. Afr., 26, 1990, p. 131-153 ; Dieux africains et Dii Mauri, Enc. berb., XV, p. $2321-2340$.

LE GLAY M., Les syncrétismes dans l'Afrique ancienne, in F.Dunand et P. Levêque, Les syncrétismes dans les religions de l'antiquité, E.P.R.O., 1975, p. 123sqq.

BENABOUM., "Le syncrétisme religieux en Afrique du Nord », Gli Interscambi Culturali e Socio Economici fra l'Africa Settentrionale e l'Europa Mediterranea, Amalfi, 1983 (1986), p. 321-332.

JANON M., « Recherches à Lambese, III : Essais sur le temple d'Esculape ", Ant. Afr., 21, 1985, p. 35-102.

XELLA P., « Aspects du culte d'Eshmoun à Carthage ", IV Coll. Intem. sur l'Hist. et l'Arch. de l'Afrique du Nord, Strasbourg, 1988, p. 131-139.

BENSEDDIK N., Le culte d'Esculape en Afrique, Paris, 1995 (Thèse de Doctorat d'État, Paris IV).

INDEX

Mots-clés : Religion 